\title{
Vaccination Guidelines During and After the COVID-19 Epidemic in China
}

\author{
Wenzhou Yu'; Yuanqiu Li'; Jing Wu'; Fuzhen Wang'; Ning Wen'; Lance Rodewald'; Lixin Hao'; Yixing Li'; \\ Hui Zheng'; Keli $\mathrm{Li}^{1}$; Chao $\mathrm{Ma}^{1}$; Dan $\mathrm{Wu}^{1}$; Lei $\mathrm{Cao}^{1}$; Lingsheng Cao'; Jiakai Ye'; Yanmin Liu'; \\ Guomin Zhang'; Wen $\mathrm{Du}^{1}$; Zhijie An ${ }^{1}$; Huaqing Wang'; Zundong Yin ${ }^{1, *}$
}

In late December 2019, clinicians in Hubei Province noticed and reported to health authorities a cluster of cases of pneumonia of unknown etiology (PUE) that turned out to be the start of the coronavirus disease 2019 (COVID-19) pandemic (1). By January 29, 2020, all provincial-level administrative divisions (PLADs) across the country had launched their highest public health emergency responses (Level 1 responses) (2-3). Vaccination service delivery was impeded by social distancing measures and restrictions on gatherings of people required in the Level 1 response protocols. Although vaccination services continued to be available in some areas, and four vaccines - rabies vaccine for postexposure prophylaxis, the birth doses of hepatitis $\mathrm{B}$ vaccine and bacille calmette-guerin (BCG) vaccine, and tetanus toxoid for wound prophylaxis continued to be administered on time in all areas, most vaccination services were stopped as part of the response (4-6). On February 3, China CDC published interim guidelines for vaccinations during the COVID-19 epidemic based on local epidemiological circumstances ( 7 ).

In early March 2020, the COVID-19 epidemic in PLADs outside of Hubei Province was coming under control, and in mid-march, China's National Health Commission issued a notice to resume routine vaccination services in an orderly manner (8). Eighty percent of the country's vaccination venues (China CDC, unpublished data) had suspended immunization services for up to two months for vaccines other than BCG, the first dose of hepatitis B, rabies, and tetanus toxoid for wound management. China CDC developed guidelines for resumption of routine immunization services and catch-up vaccinations for children who missed or delayed vaccine doses due to COVID-19. We describe the China CDC guidelines for during and after the COVID-19 epidemic in
China.

\section{VACCINATION SERVICES DURING THE COVID-19 EPIDEMIC}

During the COVID-19 epidemic, community transmission of the COVID-19 virus increased risk of infection in gatherings of people including in vaccination clinic settings. Considering the risk of COVID-19 infection and the risk of vaccine preventable diseases, China CDC made the following guidelines $(7,9)$.

1) Hepatitis $B$ vaccine and BCG vaccine should be administered to newborn infants in birth hospitals and centers on time in accordance with routine National Immunization Program recommendations. The second and third doses of hepatitis B vaccine for infants whose mothers were hepatitis B surface antigen positive should be administered on time at vaccination clinics.

2) Vaccines for post-exposure prophylaxis (PEP), e.g. rabies vaccines and tetanus toxoid, should be administered on time based on standard PEP guidelines. Patients should go to the nearest medical institution with a dog-related injury treatment clinic for timely administration of rabies PEP.

3) If COVID-19 virus is not circulating in a community (urban and rural communities and villages under the jurisdiction of sub-district offices or township people's governments), individuals can receive vaccination services during clinic operation times in accordance with local health authorities or local CDC guidelines.

4) If the COVID-19 virus is circulating in a community, administration of vaccines other than the urgent vaccinations described above can be suspended. Attention must be given to ensuring that children can be caught up on vaccinations as soon as possible once community circulation of the COVID-19 virus has ended. 
5) Individuals seeking vaccination services should make an appointment with their vaccination clinic through the Internet or by telephone. Appointments allow clinics to minimize the number of children and parents waiting to receive vaccinations or waiting to be released from observation after vaccination.

6) All people coming to a vaccination clinic should ensure they do not have a fever or other illness symptom. Children with symptoms or signs of illness will not be vaccinated. Children must be accompanied by their parents or guardians, who must also not have symptoms or signs of illness.

7) At home, following vaccination, the child's physical condition should be monitored. If the child feel hot or feverish then their temperature should be taken. Post-vaccination adverse reactions like fever and local swelling will generally resolve without treatment. If there are symptoms other than mild, local reactions or fever that can be relieved, medical advice should be sought in a timely manner.

8) To learn about personal protective measures for going to vaccination clinics, please refer to the "Provisional Guidelines for Public Medical Care During the COVID-19 Pandemic" issued by China CDC (10).

\section{CATCH-UP VACCINATION AFTER COVID-19 EPIDEMIC CONTROL}

To reduce risk of COVID-19 virus transmission during the epidemic, most vaccination clinics in China suspended vaccination services for vaccines other than BCG, hepatitis B vaccines, rabies, and tetanus toxoid, as described above. As the level of control over the COVID-19 pandemic in China improves, local vaccination clinics should resume routine immunization services and provide catch-up vaccinations based on the following technical guidelines.

\section{Premises}

Vaccination clinics should consider requirements and anticipated staffing needs for COVID-19 prevention and control efforts as they arrange vaccination services $(8)$.

In counties and districts where there have been no cases or all imported cases have been controlled (i.e. no new confirmed or suspected infections within 14 days after the last case and close contacts have been released from quarantine), vaccination clinics should restart routine immunization and implement catch-up vaccination in an orderly manner.

\section{Who should or should not receive catch- up vaccinations?}

1) Anyone whose vaccinations have been postponed due to the COVID-19 pandemic;

2) Anyone who missed any vaccinations or is not completely up-to-date on vaccinations based on the National Immunization Program schedule;

3) Appointments and catch-up immunization shall be suspended for individuals in the following circumstances:

Anyone confirmed to have COVID-19 and asymptomatic infected individuals who have not been quarantined for 14 days after leaving the hospital; those who have come into contact with someone infected with COVID-19 virus within 14 days; anyone who has been present in an epidemic area within 14 days or whose family members have had symptoms like fever and cough within 14 days.

\section{Catch-up vaccination principles and procedures}

Principles and guidelines for catch-up immunization Vaccines that are covered by the National Immunization Program including hepatitis B, BCG, polio, MMR, DTaP, Japanese encephalitis, meningococcal, and hepatitis A vaccines, shall be considered priority vaccines. Vaccines that are not covered by the National Immunization Program should be caught up in a timely manner. Program and non-program vaccines can be administered together in the same clinic visit.

Individuals who have missed doses prescribed by the National Immunization Program schedule only need to complete the missed doses; there is no need to restart the vaccination series for any vaccine.

Individuals who have missed vaccines prescribed by the National Immunization Program should complete the vaccination schedule based on their age and vaccination procedures, including minimum vaccination intervals and number of doses described in the catch-up immunization section.

If it is not feasible to complete a vaccination series with vaccines from the same manufacturer, the same vaccine type from a different manufacturer can be administered instead.

Catch-up immunization of vaccines covered by the National Immunization Program 
Hepatitis $\boldsymbol{B}$ vaccine. Newborns who were not vaccinated within 24 hours of birth should receive their first dose of hepatitis $B$ vaccine as an urgent priority. Newborns whose mothers are hepatitis B surface antigen positive must receive the second and third doses in a timely manner. The interval between the first and second dose should be at least 28 days, and the interval between the second and third dose should be at least 60 days.

BCG vaccine. Newborns who did not receive BCG vaccine within 24 hours of birth should be vaccinated as a priority. Infants below 3 months of age who did not receive the BCG vaccine can be vaccinated immediately; children between 3 months and 3 years who have tested negative for tuberculin pure protein derivatives (TB-PPD) or BCG protein derivatives (BCG-PPD) shall receive a catch-up vaccination; children older than 4 years do not need to receive catch-up BCG vaccination.

Polio vaccine. Children younger than 4 years old should receive 3 doses of polio vaccine, and those who are 4 years or older should receive 4 doses. IPV should be administered first, followed by bOPV. Children born after October 1, 2019 should receive IPV for the first 2 doses, and bOPV for the second 2 doses.

$D T a P$ and DT. Children aged between 3 months and 5 years old who have not received DTaP vaccine should complete 4 doses of DTaP vaccine. The interval between the first 3 doses should be at least 28 days, and the interval between the third dose and the fourth dose should be at least 6 months. Children aged 6 years or older who have received less than 3 doses of DTaP and DT vaccines should receive DT vaccine to complete 3 doses, children aged between 6 and 11 years old should receive adsorbed diphtheria and tetanus combined vaccines (for children), while children aged 12 years or older should receive adsorbed diphtheria and tetanus combined vaccines (for adults and adolescents). The interval between the first dose and the second dose should be 1-2 months, and the interval between the second dose and the third dose should be 6-12 months.

Measles and rubella vaccine and MMR vaccine. Children aged at least 8 months old should receive measles and rubella vaccine or MMR vaccines (to be selected according to the recommendations of the local immunization program); and children aged at least 18 months old should receive the MMR vaccine. Children aged 24 months and older who have not received 2 doses of measles-containing vaccines shall receive measles and rubella vaccines or MMR vaccine. The interval between the two doses shall be at least 28 days.

Japanese encephalitis vaccine. Children who have not received Japanese Encephalitis vaccines according to the national immunization schedule and who are aged less than 15 years old should receive 2 doses of live attenuated Japanese Encephalitis vaccines with an interval of at least 12 months between doses; or 4 doses of inactivated Japanese Encephalitis vaccines. For inactivated JE vaccine, the interval between the first dose and the second dose should be 7-10 days, the interval between the second dose and the third dose should be 1-12 months, and the interval between the third dose and the fourth dose should be at least 3 years.

Group $A$ meningococcal polysaccharide vaccine and group $A+C$ meningococcal polysaccharide vaccine. Children who have not received group A meningococcal polysaccharide vaccines and are aged less than 24 months of age should receive 2 doses of group A meningococcal polysaccharide vaccines with an interval of at least 3 months between doses; children aged 24 months or older should receive 2 doses of group $\mathrm{A}+\mathrm{C}$ meningococcal polysaccharide vaccines with an interval of at least 3 years between doses. No group A meningococcal polysaccharide vaccines will be administered as catch-up doses. The interval between the first dose of group $\mathrm{A}+\mathrm{C}$ meningococcal polysaccharide vaccines and the second dose of group $\mathrm{A}+\mathrm{C}$ meningococcal polysaccharide vaccines should be at least 12 months.

Hepatitis $A$ vaccine. Children aged older than 24 months who have not received Hepatitis A vaccine should receive 1 dose of attenuated live hepatitis $A$ vaccine or 2 doses of inactivated hepatitis $A$ vaccines with an interval of at least 6 months between doses of inactivated hepatitis A vaccine. Children who have received the first dose of inactivated hepatitis A vaccines but are unable to receive the second dose of inactivated hepatitis $A$ vaccines can receive 1 dose of attenuated live hepatitis A vaccines with an interval of at least 6 months between the inactivated vaccine dose and the live vaccine dose.

Catch-up immunization of vaccines not covered by National Immunization Program

After prioritization of vaccines that are covered by the National Immunization Program, vaccine recipients whose non-covered vaccinations have been postponed are encouraged to complete all subsequent doses using the same category of non-covered vaccines.

If a vaccination is postponed due to the COVID-19 epidemic, and the age of vaccine recipients exceeds the 
limits set out in vaccine package inserts, vaccinations can still be completed with informed consent of the vaccine recipient or their guardians with the exception of oral rotavirus vaccine. If the recipients or their custodians do not consent, the vaccination should be cancelled.

\section{Technical requirements of catch-up immunization \\ Preparation before vaccination}

Local vaccination clinics can work with rural government officials, sub-district offices (resident committees) and community service centers, as well as kindergartens and school teachers, to inform parents and supervise vaccination efforts through the epidemic joint prevention and control mechanism.

Vaccination clinics should increase the number of vaccination doctors and nurses, appropriately increase the workdays for vaccination, and provide vaccination appointment services. By consolidating the vaccination information of recipients, vaccination clinics should accurately identify individuals who have delayed or missed a vaccination, and arrange catch-up immunization through telephone, SMS, or WeChat based on the service area of vaccination clinics and number of vaccination doctors and nurses.

Vaccination clinics should reduce the number of persons accompanying vaccine recipients, check the health conditions of the vaccine recipients and the persons accompanying them, and inquire, inspect, and report their health conditions before they enter the vaccination clinics as required by local health authorities.

Vaccination clinics shall clean, ventilate, and disinfect the internal environment appropriately.

\section{Specific measures during vaccination}

Based on local regulations on COVID-19 prevention and control, vaccination doctors and nurses shall use personal protective equipment, and wear surgical masks, work clothes, caps, and gloves as recommended.

Vaccination doctors and nurses shall strictly implement verification procedures and confirm the consistency of information on vaccination certificates and vaccine packages before administering vaccines.

Vaccination doctors and nurses shall disinfect their hands with hand sanitizers before administering vaccines.

\section{Treatment after vaccination}

After administering vaccines, vaccination doctors and nurses shall disinfect their hands again with hand sanitizers.

After administering vaccines, vaccination doctors and nurses shall make appointments with vaccine recipients or their guardians for upcoming vaccinations. Vaccine recipients should stay for an observation period of 30 minutes while avoiding crowded areas.

\section{Evaluation of catch-up immunization \\ Goals}

The catch-up immunization should be completed within 2 months with a completion rate of at least $95 \%$ for vaccines covered by the National Immunization Program.

Investigation and registration of individuals who have missed vaccination should be completed within 2 months with a completion rate of at least $90 \%$ for vaccines covered by the National Immunization Program.

The vaccination rate of vaccines covered by the National Immunization Program should be at least $90 \%$.

\section{Evaluation methods and data reporting}

After the COVID-19 epidemic has stabilized for 2 months, 2-3 counties from every city, as well as 1 community and 2 township from every county should be selected at random for evaluation of catch-up immunization to ensure that the catch-up immunization has achieved the expected results.

Existing immunization information systems can be used to evaluate catch-up immunization, and places without such systems can implement on-site surveys.

Within one month after catch-up immunization, local vaccination clinics should analyze their catch-up immunization data, draft their reports, summarize the data at different levels and submit the reports to their upper-level disease control agencies and health authorities.

doi: $10.46234 / \mathrm{ccdcw} 2020.169$

\# Corresponding author: Zundong Yin, yinzd@chinacdc.cn.

${ }^{1}$ National Immunization Program, Chinese Center for Disease Control and Prevention, Beijing, China.

Submitted: April 15, 2020; Accepted: June 03, 2020

\section{REFERENCES}

1. Li Q, Guan XH, Wu P, Wang XY, Zhou L, Tong YQ, et al. Early transmission dynamics in Wuhan, China, of novel coronavirus infected pneumonia. N Engl J Med 2020;382:1199-207. http://dx.doi.org/10.1056/NEJMoa2001316. 
2. National Health Commission. China is responding to the outbreak of pneumonia caused by novel coronavirus. https://mp.weixin.qq.com/ s/wo8NqLsBcMVNw4X5iz31VA. [2020-01-21]. (In Chinese)

3. National Health Commission. The National health commission worked with relevant departments to prevent and control the outbreak of pneumonia caused by novel coronavirus. http://www.nhc.gov.cn/yjb/ s7860/202001/d9570f3a52614113ae0093df51509684.shtml. [202001-21]. (In Chinese)

4. Suk JE, Jimenez AP, Kourouma M, Derrough T, Baldé M, Honomou $P$, et al. Post-ebola measles outbreak in Lola, Guinea, January-June 2015(1). Emerg Infect Dis 2016;22(6):1106 - 8. http://dx.doi.org/10. 3201/eid2206.151652.

5. World Health Organization. COVID-19: Strategic planning and operational guidance for maintaining essential health services during an outbreak. https://apps.who.int/iris/handle/10665/331561?search-result $=$ true\&query=COVID-19\%3A+Operational+guidance+for+maintaining + essential + health+services + during + an + outbreak \&scope $=\% 2 \mathrm{~F} \& \mathrm{rpp}=10 \&$ sort_by=score\&order=desc. [2020-03-25]

6. World Health Organization. Vaccination in acute humanitarian emergencies: a framework for decision making. https://www.who.int/ immunization/documents/who_ivb_17.03/en/. [2020-03-25]

7. Chinese Center for Disease Control and Prevention. Provisional guidelines for vaccination during the COVID-19 pandemic. https://mp.weixin.qq.com/s/LLdDUKzUPPopCUK-0EsxAw. [202002-3]. (In Chinese)

8. National Health Commission. Notice on coordinating the prevention and control of COVID-19 epidemic and carrying out vaccination in a comprehensive and orderly manner. http://www.nhc.gov.cn/jkj/s3581/ 202003/c195220e8ece4208b0b30b2a6a0433ce.shtml. [2020-03-17]. (In Chinese)

9. Professional Committee on Vaccines and Health. Guiding Principles Vaccination during the COVID-19 Pandemic. https://mp.weixin. qq.com/s/-nMs0cHNVDDhZLGt10BP0g. [2020-02-3]. (In Chinese)

10. Chinese Center for Disease Control and Prevention. Provisional guidelines for public medical care during the COVID-19 pandemic. https://mp.weixin.qq.com/s/KpuGzeNqv5LDG6n_CnO2xw. [202002-11]. (In Chinese) 